\title{
AVALIAÇÃO DA EFICÁCIA DE CREMES DENTAIS CLAREADORES COM USO ASSOCIADO OU NÃO DE ESCOVA DENTAL ESPECIAL
}

\author{
AN EVALUATION OF THE WHITENING TOOTHPASTES' EFICACY \\ WITH OR WITHOUT THE ASSOCIATION OF SPECIAL TOOTHBRUSHES
}

\author{
Joanna Paula Freire de Lima Silva \\ Marília Cunha de Paiva Netto \\ Renata Pedrosa Guimarães
}

Cláudio Heliomar Vicente da Silva

\section{Resumo}

Avaliou-se o efeito do uso de cremes dentais clareadores, associados ou não a escova dental especial, sobre a cor dos dentes. Sessenta e quatro pacientes foram aleatoriamente divididos em grupos segundo o tratamento clareador: G1A-flúor gel neutro (controle negativo); G1B - peróxido de carbamida a $10 \%$ (controle positivo); G2 - Colgate ${ }^{\circledR}$ Branqueadora; G3 - Malvatricin Branqueadora; G4 - creme dental clareador manipulado - Phormula Ativa). Os grupos experimentais foram subdivididos em subgrupos: A - utilização de escova dental convencional Oral B $₫$ Indicator plus; $\mathrm{B}$ - uso de escova especial Oral $\mathrm{B}{ }^{\circledR}$ Advanced Ártica. As alterações cromáticas observadas inicialmente e após quatro semanas com uso do espectrofotômetro digital não revelaram diferenças significativas ( $p>0,05$ - Teste de Mann-Whitney) quando comparados os grupos experimentais com o Grupo Controle Negativo, mas revelou diferença quando comparado ao Grupo Controle Positivo. A comparação entre as escovas dentais utilizadas não revelou diferenças estatísticas. Concluiu-se que os cremes dentais avaliados não foram capazes de produzir efeito clareador semelhante ao clareamento supervisionado; o uso da escova dental especial não exerceu influência sobre o efeito clareador de nenhum dos produtos utilizados.

Palavras-chave: Clareamento de Dente - CREME DENTAL - ESCOVAÇÃO DENTÁRIA - ESTÉTICA.

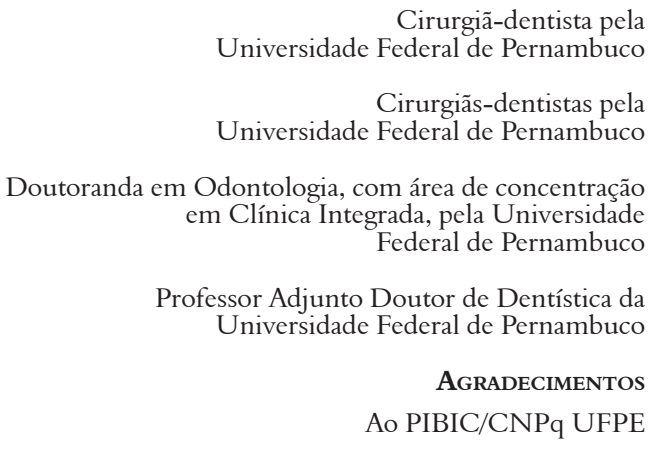

Abstract

This study has evaluated the bleaching effect of whitening toothpastes associated or not to special toothbrushes. Sixtyfour patients were randomly divided into groups according to the bleaching treatment used (G1A: neutral fluoride gel; G1B: Carbamide peroxide 10\%; G2: Colgate () Branqueadora; G3: Malvatricin Branqueadora; G4: manipulated whitening toothpaste - Phormula Ativa). The experimental groups were divided into subgroups A: using conventional toothbrush Oral B Indicator ${ }^{\circledR}$ plus; $B$ : using

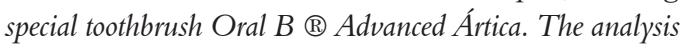
of the difference of color observed initially and after four weeks of treatment using the Mann-Whitney test did not show significant differences $(p>0.05)$ when the experimental groups were compared to the Negative Control Group, but it showed differences when compared to the Positive Control Group. The comparison between the toothbrushes revealed no statistical differences. One may conclude that the evaluated toothpastes were not effective in producing a whitening effect similar to the one obtained through supervised whitening; the use of a special toothbrush did not influence the whitening effect of any of the products used.

KEYWORDS: TOOTH BLEACHING - TOOTHPASTE TOOTH BRUSHING - ESTHETICS. 


\section{INTRODUÇÃO}

Vive-se em uma sociedade visual na qual a expressão facial é um elemento muito importante no processo de comunicação. Por isso, o significado dos dentes em um sorriso, e de um sorriso em uma face, não deve ser subestimado. Um sorriso saudável não apenas influencia a aparência física de uma pessoa, como também melhora sua autoestima, que por sua vez tem importância crucial nos processos de relacionamento. ${ }^{1}$

$\mathrm{Na}$ verdade, o senso comum percebe nos dentes claros a existência de um padrão estético de beleza em Odontologia, ${ }^{2,3}$ por isso a cor dos dentes tem levado muitos pacientes aos consultórios dentários em busca de dentes cada vez mais brancos. ${ }^{4}$

Para atender a esta demanda, as técnicas de clareamento têm sido modificadas para a obtenção de resultados cada vez mais satisfatórios. Além das técnicas realizadas pelos profissionais, o mercado vem oferecendo vários produtos com o intuito de promover clareamento, como cremes, géis dentais, fitas e esmaltes branqueadores. ${ }^{3}$

Inúmeros tipos de cremes dentais que propõem efeito branqueador prático e rápido vêm sendo ofertados. ${ }^{5}$ No entanto, esses dentifrícios parecem apresentar eficiência limitada, uma vez que contêm apenas abrasivos para remoção de manchas da superfície externa dos dentes. Somente alguns desses produtos contêm agentes químicos clareadores que poderiam justificar sua eficácia. ${ }^{6}$

O marketing industrial veicula na mídia um número cada vez maior de produtos (cremes e escovas dentais) disponíveis e acessíveis à população, apresentando apelos clareadores, que despertam a atração dos consumidores. Por isso, este estudo objetivou avaliar clinicamente o efeito do uso de cremes dentais clareadores, associados ou não, a escova dental especial, sobre a cor dos dentes.

\section{REVISÃo DA LITERATURA}

As alterações cromáticas que acometem a estrutura dentária podem ter uma origem intrínseca ou extrínseca. As intrínsecas envolvem alterações congênitas, que ocorrem durante a fase de formação do elemento dentário, ou adquiridas, que podem ser provocadas por iatrogenia ou traumatismo. Já as alterações extrínsecas são manchas superficiais resultantes principalmente de substâncias corantes ingeridas em excesso pelo paciente, como fumo, café, chás, dentre outras. $4,7,8,9$

Para a reversão destas alterações, o clareamento é um procedimento estético não invasivo que evoluiu muito na última década. O desenvolvimento de novas técnicas para sua aplicação trouxe mais conforto para o paciente e novas perspectivas para o cirurgião dentista. ${ }^{10}$ Dentre as técnicas de clareamento existentes, destacam-se a técnica realizada em consultório e o clareamento supervisionado, ou caseiro. ${ }^{11}$

No clareamento supervisionado o paciente faz uso de uma moldeira personalizada, confeccionada pelo profissional em acetato ou material plástico, e deverá ser orientado quanto à forma de aplicação do agente clareador, período, frequência e tempo de uso. ${ }^{12}$ Vários estudos clínicos comprovaram que o clareamento dental caseiro com peróxido de carbamida a $10 \%$ é uma técnica simples, segura, de fácil aplicação e com resultados satisfatórios no longo prazo. ${ }^{10}$ Diante do crescente destaque da odontologia estética, a procura pelo clareamento caseiro aumentou consideravelmente, tanto pelo baixo custo quanto por sua praticidade. ${ }^{11}$

Já na técnica realizada em consultório, o profissional utiliza uma proteção gengival, que pode ser feita com barreira gengival ou isolamento absoluto, e aplica um agente clareador, à base de peróxido de hidrogênio em concentrações que variam de $35 \%$ a $37 \%$ sobre a estrutura dentária. Diversas técnicas e formas de aplicação estão disponíveis, as quais podem utilizar, ou não, energia luminosa para aceleração da reação. ${ }^{13}$

Independentemente da técnica realizada, o produto final e principal agente ativo é o peróxido de hidrogênio, o qual atua como um potente agente oxidante por meio da formação de radicais livres, moléculas de oxigênio reativo e ânions de peróxido. Estes produtos oxirredutores atacam as longas cadeias das moléculas cromóforas, responsáveis pelo escurecimento, dividindoas em moléculas mais claras e menores que se difundem mais facilmente pela estrutura dentária. O efeito clareador depende principalmente da concentração do agente clareador, da habilidade deste agente em alcançar os cromóforos, da duração e do número de vezes que a substância entra em contato com essas moléculas. ${ }^{14}$ 
No entanto, o mercado de produtos cosméticos sugere a utilização de produtos clareadores domésticos, livremente adquiridos pelos pacientes em supermercados e farmácias, em forma de cremes dentais, por exemplo, com a proposta de promover efeito clareador rápido e a custos reduzidos. Uma rápida apreciação dos ingredientes contidos nos cremes dentais clareadores revela que a maior parte é formulada para controlar manchas dentárias extrínsecas em vez de mudar a cor natural do dente por meio de uma ação branqueadora. No mercado brasileiro, os abrasivos mais usados nos dentifrícios são o carbonato de cálcio e a sílica, embora outros possam estar presentes. ${ }^{15}$ Alguns produtos contêm também outros agentes para benefício da saúde, principalmente contra cáries, gengivite e sensibilidade dentária. ${ }^{16}$

Dentifrícios branqueadores trabalham por ação química ou remoção mecânica das manchas. Pastas branqueadoras que removem manchas superficiais não devem ser confundidas com agentes clareadores que agem quebrando os pigmentos para atenuar a cor dos dentes. ${ }^{17,18}$

Pesquisas indicam que enquanto a adição de abrasivos pode facilitar o processo de polimento dental, a ação de atrição das cerdas da escova, associada a essas substâncias, pode aumentar os riscos de desenvolvimento de lesões sobre a estrutura dentária. ${ }^{6,19}$ Desse modo, o uso indiscriminado desses produtos é desaconselhável, uma vez que pode trazer danos à estrutura dentária, como o desgaste excessivo do esmalte. ${ }^{6,15}$
O crescente apelo comercial também atinge o mercado de escovas dentais, instrumento primordial para o autocuidado e o objeto mecânico universalmente mais utilizado para a higiene bucal. ${ }^{20}$ A remoção mecânica da placa é conseguida por meio de um contato direto da escova com dentes e gengiva, juntamente com a ação abrasiva de suas cerdas. ${ }^{21}$ Vários tipos de escovas com design especial têm sido desenvolvidos nos últimos anos a fim de melhorar a remoção da placa. ${ }^{22}$ Apesar dos vários designs e tipos de cerdas disponíveis atualmente, há consenso no meio odontológico em sugerir escovas dentais de cerdas macias e, preferencialmente, com pontas arredondadas, por serem consideradas essas mais compatíveis com os delicados tecidos da região intrassulcular. ${ }^{23}$

\section{Materiais E MÉTOdo}

Para a realização do presente estudo foram selecionados pacientes de ambos os gêneros, maiores de 21 anos, que apresentassem todos os dentes superiores anteriores naturais, vitalizados, escurecidos naturalmente ou fisiologicamente, sem restaurações e com cor mais escura ou igual a B2 na escala VITA® do guia de matizes; saúde bucal; ausência de sensibilidade, lesões cervicais e fraturas.

Após leitura e assinatura do Termo de Livre Consentimento, pré-aprovado pelo Comitê de Ética em Pesquisa com Seres Humanos da UFPE (registro 96.404), os pacientes selecionados foram distribuídos em quatro grupos e oito subgrupos, de acordo com as variáveis a serem pesquisadas, por meio de sorteio simples

QuAdro 1: CARACTERIZAÇÃO DOS GRUPOS E SUBGRUPOS.

\begin{tabular}{|c|c|c|c|}
\hline GRUPO & SUBGRUPO & PRODUTO & ESCOVA DENTAL \\
\hline \multirow{2}{*}{$\begin{array}{c}1 \\
\text { Controle }\end{array}$} & A- Negativo & Placebo (Flúor Gel Neutro) & $\begin{array}{l}\text { Escova convencional } \\
\text { (Oral B Indicator Plus) }\end{array}$ \\
\hline & B- Positivo & $\begin{array}{l}\text { Peróxido de Carbamida 10\% - Phormula } \\
\text { Ativa } \\
\text { (Clareamento supervisionado) }\end{array}$ & $\begin{array}{l}\text { Escova convencional } \\
\text { (Oral B Indicator Plus) }\end{array}$ \\
\hline \multirow{2}{*}{2} & A & Colgate Branqueadora & $\begin{array}{l}\text { Escova convencional } \\
\text { (Oral B Indicator Plus) }\end{array}$ \\
\hline & B & Colgate Branqueadora & $\begin{array}{l}\text { Advantage Artica } \\
\text { (Oral B) }\end{array}$ \\
\hline \multirow{2}{*}{3} & A & Malvatricin Branqueadora & $\begin{array}{l}\text { Escova convencional } \\
\text { (Oral B Indicator Plus) }\end{array}$ \\
\hline & B & Malvatricin Branqueadora & $\begin{array}{c}\text { Advantage Artica } \\
\text { (Oral B) }\end{array}$ \\
\hline \multirow[b]{2}{*}{4} & A & $\begin{array}{c}\text { Creme Dental Clareador Manipulado } \\
\text { (Phormula Ativa) }\end{array}$ & Escova convencional \\
\hline & B & $\begin{array}{l}\text { Creme Dental Clareador Manipulado } \\
\text { (Phormula Ativa) }\end{array}$ & Advantage Artica \\
\hline
\end{tabular}


Quadro 2: Produtos Clareadores E escovas aVAliadas.
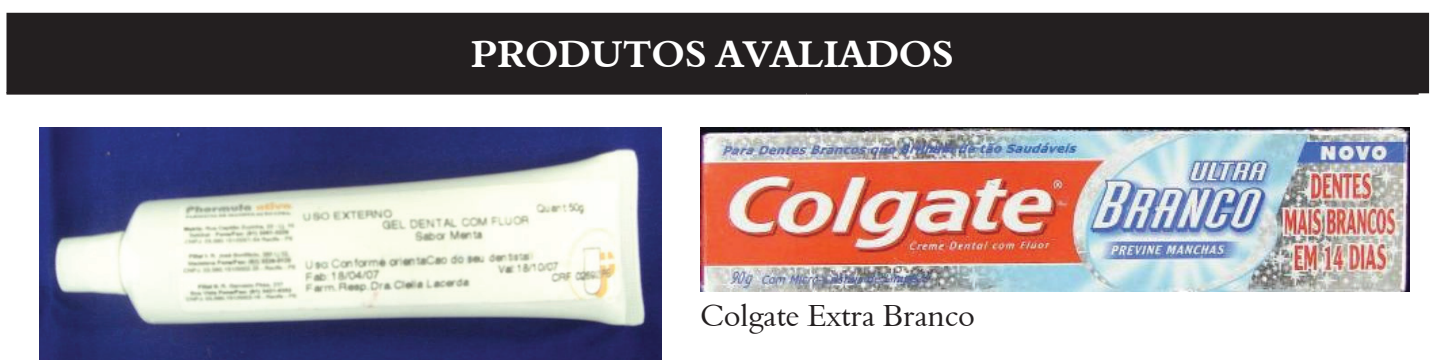

Colgate Extra Branco

Flúor Gel Neutro - Phormula Ativa
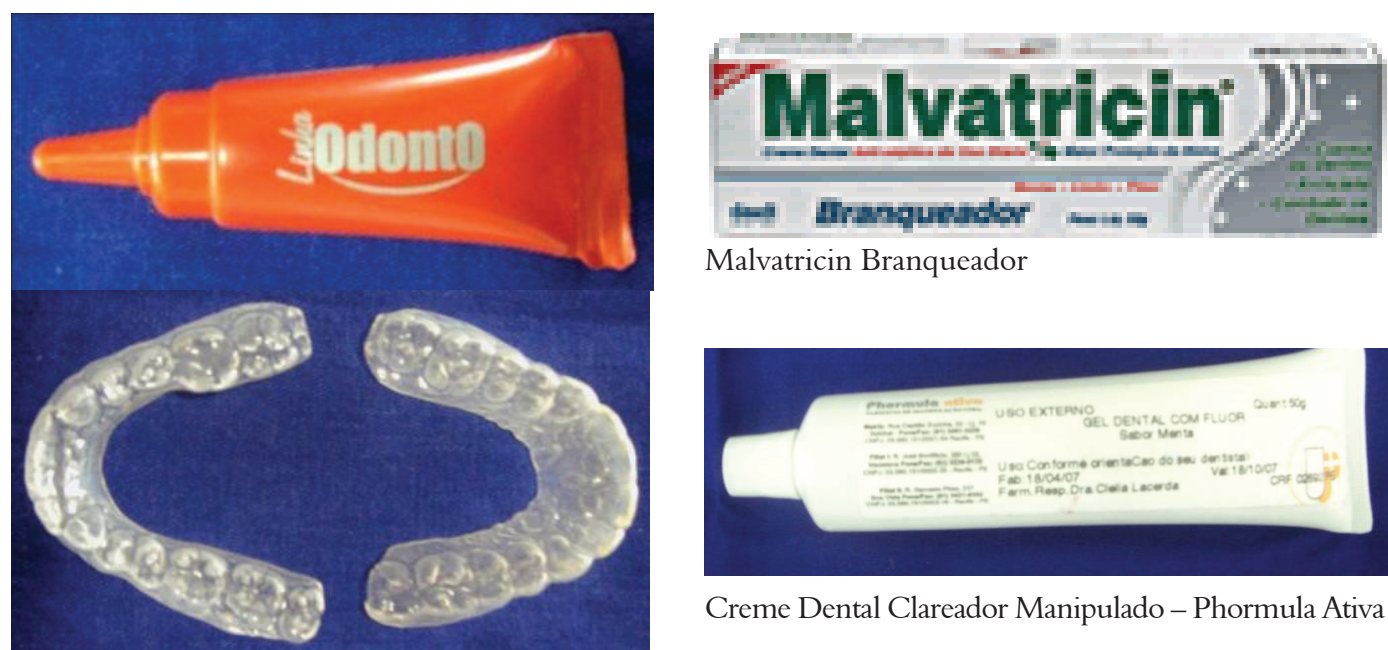

Malvatricin Branqueador

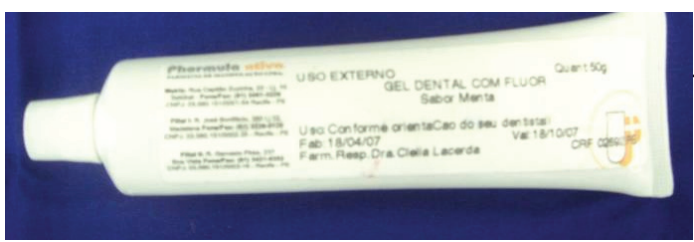

Creme Dental Clareador Manipulado - Phormula Ativa

Peróxido de Carbamida 10\% - Phormula Ativa

\section{ESCOVAS AVALIADAS}

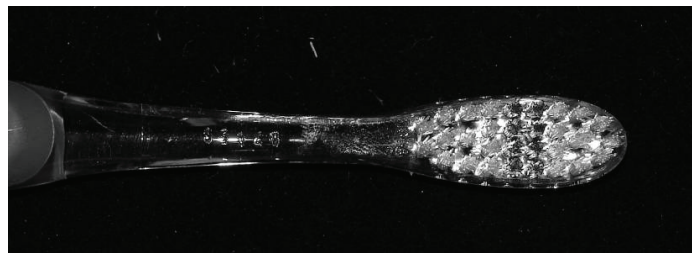

Indicator Plus- Oral B

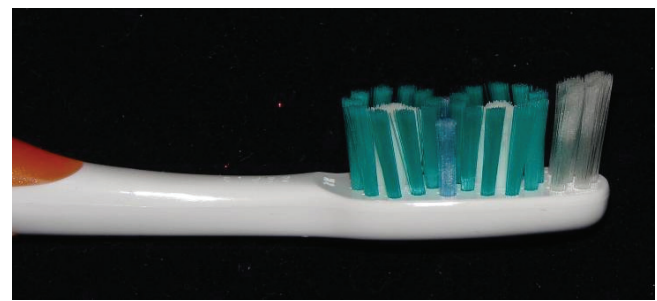

Advantage Ártica - Oral B

Sessenta e quatro pacientes receberam tratamento, por quatro semanas, de acordo com as sessões descritas no Quadro 3. Visitas semanais foram agendadas para registro das alterações cromáticas.

QuAdro 3: Metodologia CLÍNICA APLiCADA. 


\begin{tabular}{|c|c|}
\hline $1^{a}$ SESSÃO & SESSÕES SUBSEQUENTES: \\
\hline $\begin{array}{l}\text { 1. Leitura e assinatura do Termo de Consentimento. } \\
\text { 2. Anamnese. } \\
\text { 3. Exame físico. } \\
\text { 4. Preenchimento da ficha clínica. } \\
\text { 5. Profilaxia com pasta de pedra-pomes e água. } \\
\text { 6. Registro inicial da cor. } \\
\text { 7. Entrega do produto e escova. } \\
\text { 8. Orientações quanto ao uso. }\end{array}$ & $\begin{array}{l}\text { 1. Avaliação da sensibilidade transoperatória. } \\
\text { 2. Profilaxia com pedra-pomes e água. } \\
\text { 3. Registro da cor. } \\
\text { 4. Orientações quanto à higiene bucal. }\end{array}$ \\
\hline
\end{tabular}

Os pacientes do subgrupo 1B (controle positivo) realizaram a técnica de clareamento supervisionado, portanto o tratamento foi diferenciado dos demais. Na primeira sessão clínica, além dos procedimentos já descritos, foi realizada moldagem superior e inferior com alginato (Jeltrate/ Dentsply) e confecção de modelos em gesso pedra tipo III (Diamante) para confecção da moldeira de acetato personalizada feita no plastificador a vácuo. Na segunda sessão clínica, a moldeira personalizada foi entregue juntamente com o agente clareador (peróxido de carbamida 10\%) e foram dadas as orientações quanto ao uso e cuidados durante o tratamento, o qual consistiu do uso da moldeira por oito horas diárias, durante o sono, por quinze dias. Os demais procedimentos seguiram o protocolo geral.

As leituras das modificações cromáticas foram realizadas antes do tratamento clareador e após cada semana com o espectrofotômetro digital portátil (EasyShade - Vita).

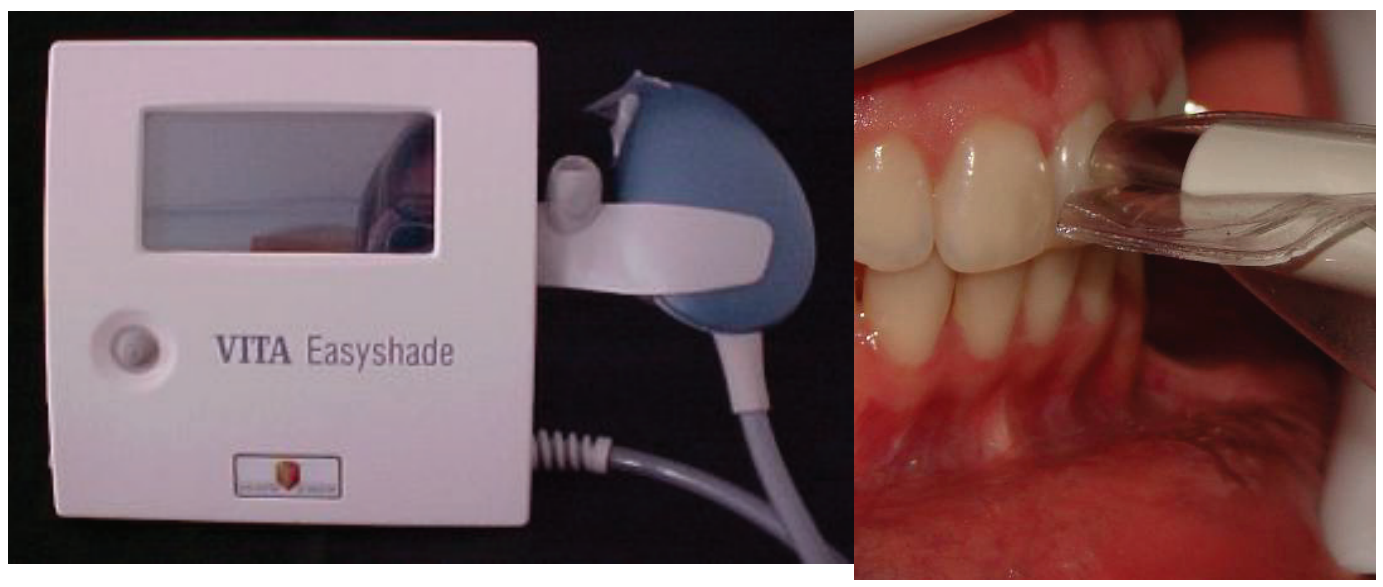

Figura 1: EsPedtrofotôMETRO DigitAL (EASYSHADE - VITA).

Foram medidos os terços médios vestibulares dos seis dentes antero-superiores (de canino a canino).

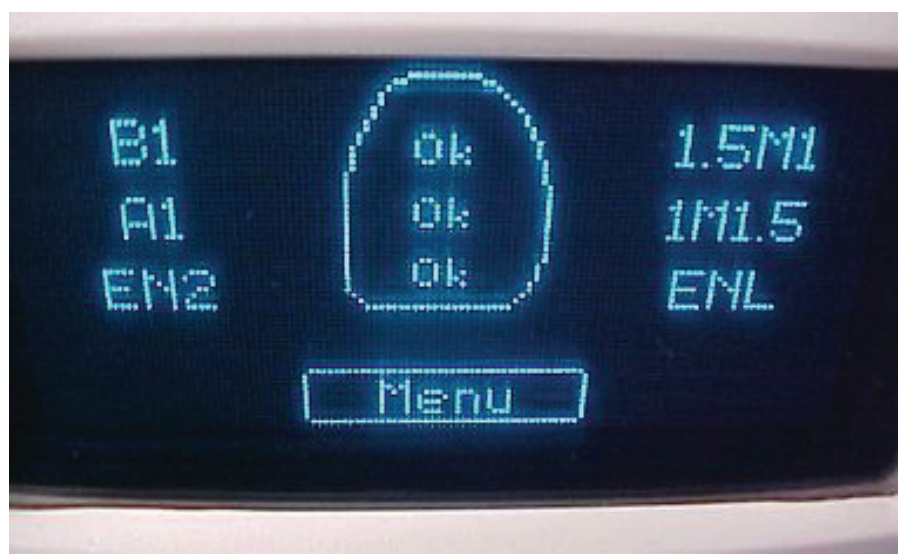

Figura 2. Valores Fornecidos pelo EasyShade durante a mensuração. A - Escala Vita® Clássica. B - Escala Vita® 3D Máster. 


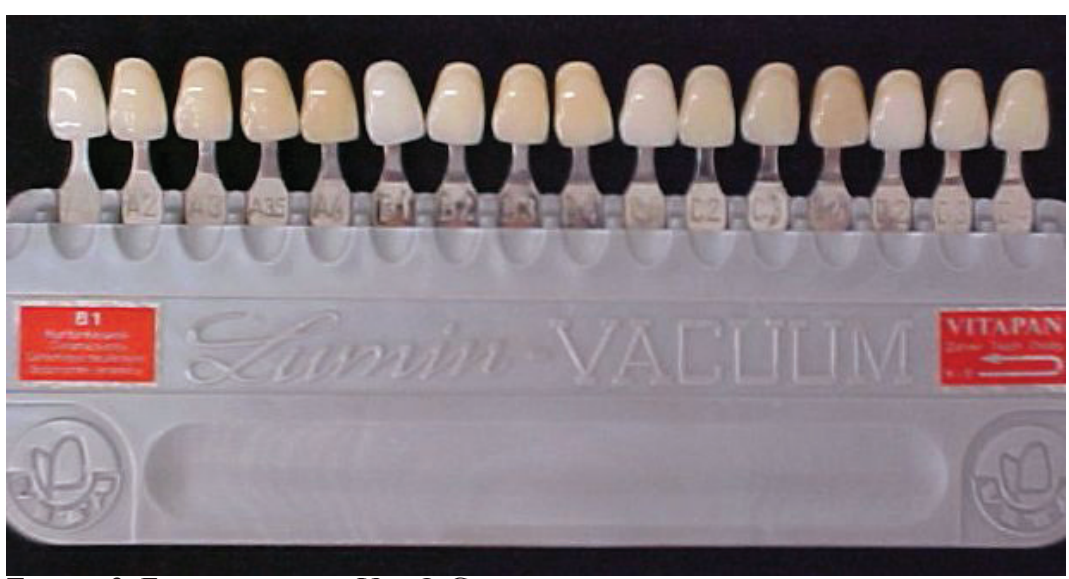

Figura 3. Escala dE CORES Vita® ClásSicA.

Os valores obtidos com o Easy Shade / Vita (Figura 2), que são baseados na escala Vita Clássica (Figura 3), foram convertidos para uma escala numérica a fim de facilitar as manobras estatísticas, considerando a média de coloração dos seis dentes ântero-superiores.

Quadro 4. Guia de Matizes: conversão de valores da Escala de cores Vita® Clássica para valores NUMÉRICOS.

Claro

Escuro

\begin{tabular}{|c|c|c|c|c|c|c|c|c|c|c|c|c|c|c|c|}
\hline B1 & A1 & B2 & D2 & A2 & C1 & C2 & D4 & A3 & D3 & B3 & A3,5 & B4 & C3 & A4 & C4 \\
\hline 1 & 2 & 3 & 4 & 5 & 6 & 7 & 8 & 9 & 10 & 11 & 12 & 13 & 14 & 15 & 16 \\
\hline
\end{tabular}

QuAdro 5. Formulário para REgistro dos DADOS.

\begin{tabular}{|c|c|c|c|c|c|c|c|c|c|c|}
\hline DENTES & $\begin{array}{l}\text { VALOR } \\
\text { DATA: }\end{array}$ & $\begin{array}{l}\text { NÚMERO } \\
\text { INICIAL }\end{array}$ & $\begin{array}{c}\text { VALOR } \\
1^{\text {a }} \text { Sem } \\
\text { DATA: }\end{array}$ & $\begin{array}{c}\text { NÚMERO } \\
1^{\mathrm{a}} \mathrm{Sem}\end{array}$ & $\begin{array}{l}\text { VALOR } \\
2^{\mathrm{a}} \text { Sem } \\
\text { DATA: }\end{array}$ & $\begin{array}{c}\text { NÚMERO } \\
2^{\mathrm{a}} \mathrm{Sem}\end{array}$ & $\begin{array}{l}\text { VALOR } \\
3^{\text {a }} \text { Sem } \\
\text { DATA: }\end{array}$ & $\begin{array}{c}\text { NÚMERO } \\
3^{\text {a }} \mathrm{Sem}\end{array}$ & $\begin{array}{c}\text { VALOR } \\
4^{a} \text { Sem } \\
\text { DATA: }\end{array}$ & $\begin{array}{l}\text { NÚMERO } \\
4^{\mathrm{a}} \mathrm{Sem}\end{array}$ \\
\hline 13 & & & & & & & & & & \\
\hline 12 & & & & & & & & & & \\
\hline 11 & & & & & & & & & & \\
\hline 21 & & & & & & & & & & \\
\hline 22 & & & & & & & & & & \\
\hline 23 & & & & & & & & & & \\
\hline UÉDIA & & & & & & & & & & \\
\hline
\end{tabular}

\section{Resultados e Discussão}

Dentre os 64 pacientes selecionados para este estudo, quatro desistiram. Assim, a análise estatística dos dados considerou um total de 60 indivíduos (93,7\%). Destes, 65,5\% eram do gênero feminino e $34,5 \%$, do gênero masculino. A média de idade da amostra foi de 23,1 anos.

Considerando a avaliação da eficácia dos cremes dentais clareadores, a Tabela 1 apresenta a comparação entre os valores médios da diferença entre a coloração inicial e final dos elementos dentários para cada grupo. 
TABELA 1. MÉdia DAS DIFERENÇAS ENTRE A COLORAÇÃO INICIAL E FINAL E O DESVIO-PADRÃO PARA CADA GRUPO.

\begin{tabular}{cccc}
\hline GRUPO & MÉDIA * & N & DESVIO-PADRÃO \\
\hline 1A & $0,3340(\mathrm{a})$ & 5 & 0,57 \\
1B & $3,4800(\mathrm{~b})$ & 9 & 0,49 \\
2A & $0,5838(\mathrm{a})$ & 8 & 0,72 \\
2B & $0,7386(\mathrm{a})$ & 7 & 0,50 \\
3A & $0,8563(\mathrm{a})$ & 8 & 1,14 \\
3B & $0,9743(\mathrm{a})$ & 7 & 0,41 \\
4A & $0,8125(\mathrm{a})$ & 8 & 1,16 \\
4B & $0,9175(\mathrm{a})$ & 8 & $\mathbf{1 , 4 5}$ \\
\hline TOTAL & $\mathbf{1 , 1 7 2 3}$ & $\mathbf{6 0}$ & \\
\hline
\end{tabular}

a. Letras diferentes entre parênteses demonstram a diferença estatística $(\mathrm{p}<0,05)$

* Por meio do teste Mann-Whitney, que compara a soma dos postos.

A comparação entre o Controle Negativo (1A), no qual foi utilizado somente um placebo, e o Controle Positivo (1B), no qual foi utilizada a técnica do clareamento supervisionado com peróxido de carbamida a $10 \%$, revelou diferença estatística $(p=0,001)$, reafirmando a eficácia do clareamento supervisionado, $, 10,11,12$ bem como a legitimidade dos Grupos 1A e 1B como parâmetros para avaliação da eficácia dos demais grupos.

Não foram observadas diferenças significativas entre cada grupo experimental e o Grupo Controle Negativo, independentemente da utilização da escova dental especial (Advantage/ Oral B). Por outro lado, a comparação entre todos os grupos experimentais, independentemente da utilização da escova especial, com o Grupo Controle Positivo revelou diferenças estatísticas, com os melhores resultados para o clareamento supervisionado. Estes dados demonstram que os cremes dentais clareadores avaliados não foram eficazes em promover alteração de cor significativa.

Os achados desta pesquisa corroboram os resultados obtidos por outros estudos ${ }^{6}$, ${ }^{15,18}$ que discordam do potencial dos cremes dentais clareadores para realmente reduzir o croma da estrutura dentária.

A eficácia clínica de cremes dentais clareadores, observada pelos estudos de
Nathoo et al. ${ }^{24}$ e Hoic et al., ${ }^{25}$ de acordo com suas afirmações, restringiu-se a maior remoção de manchas extrínsecas, ou seja, em virtude da maior abrasividade destes produtos sobre a estrutura dentária, e não por um efeito clareador real. Outros autores $^{6,5,15,18,19}$ também alertaram para o potencial abrasivo de cremes dentais ditos "clareadores" - potencial este comprovado não apenas pela análise de composição como também por estudos experimentais.

Por outro lado, ao analisarmos o grau de reversão cromática observado pelos cremes dentais Malvatricin Branqueador e Phormula Ativa (Tabela 1), com redução próxima a 01 tom na escala de cores e associada à menor quantidade de abrasivos que eles apresentam, estes podem ter seu uso justificado nos casos de manutenção de tratamento clareador, desde que de forma restrita (por período de uso não prolongado) e supervisionada pelo cirurgião-dentista. ${ }^{26}$

A comparação do efeito clareador obtido entre os cremes dentais testados entre si não revelou diferenças estatisticamente significativas para um nível de significância de 5\%.

E quanto à escova dental especial? Que tipo de influência foi observada sobre 
o efeito clareador? A hipótese, levantada neste trabalho e sugerida pelo fabricante, reforçava a ideia de que a presença de cerdas polidoras, dispostas de maneira circular, poderia potencializar a ação dos cremes dentais testados pelo fato de eles permanecerem mais tempo em contato com os dentes, comparado às escovas convencionais. Apesar de a análise descritiva dos dados apresentados na Tabela 1 revelar uma maior eficácia do grupo que realizou as escovações com a escova Advantage (Oral B) e uma tendência para tal afirmação, a análise estatística não revelou diferenças significativas $(p>0,05)$ ao cruzar os dados dos subgrupos A e B, independentemente do creme dental utilizado.

Apenas 12 pacientes (20\%) relataram a ocorrência de sensibilidade transoperatória grau leve. Porém 11,6\% $(n=7)$ destes já relatavam o mesmo grau de sensibilidade antes do início do tratamento. A maior frequência de sensibilidade se deu no subgrupo 1B (clareamento supervisionado), onde um terço $(\mathrm{n}=3)$ dos pacientes integrantes apresentou episódios de leve sensibilidade. Este resultado concorda com outros estudos que observaram baixa frequência de sensibilidade durante a técnica de clareamento supervisionado. ${ }^{7,9}$ A incidência de dor nos demais grupos foi insignificante.

\section{Conclusões}

1. Os cremes dentais avaliados não foram eficazes em produzir um efeito clareador significativo.

2. A associação com a escova dental especial (Advantage Ártica/Oral B) não exerceu influência estatisticamente significativa sobre o efeito clareador de nenhum dos produtos utilizados, embora a análise descritiva dos dados revele uma tendência para maior eficácia do grupo que realizou as escovações com a referida escova.

\section{REFERÊNCIAS BIBLIOGRÁFICAS}

1. Busato ALS, Hérnadez PAG, Macedo RPM. Tratamento do discromatismo dental. In: Busato ALS, Hérnadez PAG, Macedo RPM. Dentística restaurações estéticas. São Paulo: Artes Médicas; 2002. p. 489-574.

2. Amorim CCS, Beatrice LCS, Vicente da Silva CH. Influência da mídia televisiva sobre o padrão estético odontológico. Odontol Clín Cient. 2006; 5(2): 163-6.

3. Vicente da Silva CH, Souza FB, Pedroza ACB. Esvaziamento dentinário Alternativa para reversão de cor em dentes tratados endodonticamente. J Bras Clin Odontol Int. 2003; 7(37): 24-7.

4. Menezes Filho PF, Barros $\mathrm{CHO}$, Noronha JAA, Melo Junior PC, Cardoso RM. Avaliação crítica do sorriso. Int J Dent 2006; 1(1): 14-9.

5. Menezes MM, Firoozmand LM, Huhtala MFR. Avaliação do desgaste superficial do esmalte escovado com dentifrícios e submetido à ação de agentes branqueadores. Cienc Odontol Bras 2003; 6(1): 44-50.

6. Mariz ALA, Fernandes JV, Guimarães RP, Silva CHV. Conhecendo os cremes dentais "clareadores". Dental Science 2007; 1(2): 93-7.

7. Melo N, Gallego GJ, Restrepo LF, Peláez A. Blanqueamiento vitaly métodos para la valoración de su eficacia y estabilidad. Rev CES Odontol. 2006; 19(2): 53-60.

8. Pinto MCGL, Monteiro GQM; Carvalho PRB; Melo GFB, Silva CHV. Manchamento por tetraciclina: como tratar? Rev Fac Odontol Porto Alegre. 2005; 46(1): 54-8.

9. Ruiz GAO, Sá FC. Clareamento caseiro em dentes vitais. Rev Gaucha Odontol 2003; 51(1): 18-22.

10. Farias VB, Höfling RTB, Carvalho AS, Bussadori SK, Bassanta AD. Clareamento dental caseiro e clareamento dental interno. Rev Gaucha Odontol. 2003; 51(4): 289-92.

11. Sanábio BS, Gonçalves GL, Vieira PAA. Análise clínica de dois tipos de moldeira usadas no clareamento exógeno. Rev Gaucha Odontol. 2006; 54(4): 308-12.

12. Conceição EM, Dillemburg, AL. Clareamento dental. In: Conceição, EN. et al. Dentística - Saúde e Estética.Cap. 4. Porto Alegre: Artmed, 2002, p. 227-47.

13. Mangani F, Pisacane C, Pujia AM. Clareamento de dentes vitais. J Clin Dent. 2001; 2(13): 5-14.

14. Dahl JE, Pallensen U. Tooth bleaching: A critical review of the biological aspects. Crit Rev Oral Biol Med. 2003; 14(4): 292-304.

15. Andrade Junior ACC, Andrade MRTC, Machado WAS, Fischer RG. Estudo in 
vitro da abrasividade de dentifrícios. Rev Odontol Univ São Paulo. 1998; 12(3): 231-6.

16. Pontefract H, Courtney M., Smith S, Newcombe RG, Addy M. Development of methods to enhance extrinsic tooth discoloration for comparison of toothpastes 1. Studies in vitro. J Clin Periodontol. 2004; 31: 1-6.

17. American Dental Association (ADA). Whitening toothpastes. J Amer Dent Assoc 2001; 32(8): 1146-1147.

18. Claydon NCA, Moran J, Bosma ML, Shirodaria S, Addy M, Newcombe R. Clinical study to compare the effectiveness of a test whitening toothpaste with a commercial whitening toothpaste at inhibiting dental stain. J Clin Periodontol. 2004; 31: 1088-91.

19. Macdonald E, North A, Maggio B, Sufi F, Mason S, Moore C et al. Clinical Study investigating abrasive effects of three toothpastes and water in a in situ model. J Dent 2010; 38: 509-16.

20. Pereira RC, Gusmão ES, Santos RL, Galdino R, Silveira RSJ, Araújo ACS. Avaliação microbiológica das cerdas de escovas dentárias. Rev Gaucha Odontol. 2005; 53(2): 85-94.

21. Graveland MP, Rosema NAM, Timmerman MF, Van Der Weijden GA. The plaque- removing efficacy of a finger brush (I-Brush). J Clin Periodontol. 2004; 31: 1084-87.

22. Zimmer S, Didner B, Roulet JF. Clinical study on the plaque-removing ability of a new triple-headed toothbrush. J Clin Periodontol. 1999; 26: 281-5.

23. Chiapinotto GA, Meller D, Santos FB. Avaliação de meios mecânicos de limpeza. Rev Gaucha Odontol. 2001; 49(3): 161-4.

24. Nathoo S, Petrone E, Devizio W, Chaknis P, Volpe AR. A Six Week Clinical Study to Compare the Stain Removal Efficacy of Three Dentifrices. J Clin Dent. 2002; 13(2): 91-4.

25. Hoic D, Dixit N, Prencipe $M$, Subramanyam R, Cameron R, Malak RA et al. The Technology Behind Colgate Simply White Toothpaste. J Clin Dent. 2004; 15(2): 37-40.

26. Araújo DB, Campos EJ, Silva LR, Araujo RPC. Lesões de esmalte dental relacionadas aos dentifrícios clareadores. R Ci Méd Biol. 2009; 8(2): 171-81.

Recebimento: 24/5/2010 Aprovação: 17/10/2011 